\section{GENETICALLY ENGINEERED TUMOR-INFILTRATING LYMPHOCYTES (CYTOTIL15) EXHIBIT IL-2-INDEPENDENT PERSISTENCE AND ANTI-TUMOR EFFICACY AGAINST MELANOMA IN VIVO}

Rachel Burga*, Mithun Khattar, Scott Lajoie, Kyle Pedro, Colleen Foley, Alonso Villasmil Ocando, Jack Tremblay, Benjamin Primack, Meghan Langley, Dan Thornton, Stanley Tam, Emily Brideau, Theresa Ross, Gwen Wilmes, Sunandan Saha, Gabriel Helmlinger, Jeremy Tchaicha, Dhruv Sethi, Michelle Ols, Gary Vanasse, Shyam Subramanian, Jan ter Meulen. Obsidian Therapeutics, Cambridge, MA, USA

Background Adoptive cell therapy with tumor-infiltrating lymphocytes (TILs) has demonstrated tremendous promise in clinical trials for patients with solid or metastatic tumors. ${ }^{1}$ However, current TIL therapy requires systemic administration of IL-2 to promote TIL survival, and IL-2-associated toxicities greatly limit patient eligibility and reduce the long-term clinical benefit of TIL therapy. ${ }^{2}{ }^{3}$ Unlike IL-2, which promotes T cell exhaustion, IL-15 maintains antigen-independent TIL persistence through homeostatic proliferation and supports CD8+ $\mathrm{T}$ cell anti-tumor activity without stimulating regulatory $\mathrm{T}$ cells. We designed genetically engineered TILs to express a regulated form of membrane-bound IL-15 (mbIL15) for tunable long-term persistence, leading to enhanced efficacy and safety for the treatment of patients with solid tumors.

Methods Obsidian's cytoDRiVE ${ }^{\mathrm{TM}}$ platform includes small human protein sequences called drug responsive domains (DRD)s that enable regulated expression of a fused target protein under control of FDA-approved, bioavailable small molecule ligands. cytoTIL15 contains TILs engineered with mbIL15 under the control of a carbonic-anhydrase-2 DRD, controlled by the ligand acetazolamide (ACZ). After isolation from tumors, TILs were transduced and expanded in vitro through a proprietary TIL expansion process. cytoTIL15 were immunophenotyped and assessed for in vitro antigen-independent survival and co-cultured with tumor cells to assess polyfunctionality and cytotoxicity. In vivo TIL persistence and antitumor efficacy was evaluated through adoptive transfer of TILs into immunodeficient NSG mice, either naïve or implanted with subcutaneous patient-derived-xenograft (PDX) tumors.

Results cytoTIL15 and conventional IL2-dependent TILs isolated from melanoma tumor samples expanded to clinically relevant numbers over 14 days. Throughout expansion, cytoTIL15 were enriched for CD8 $+\mathrm{T}$ cells and acquired enhanced memory-like characteristics, while maintaining diverse TCRV $\beta$ sub-family representation. cytoTIL15 demonstrated enhanced potency over conventional TILs, as measured by increased polyfunctionality and cytotoxicity against tumor and PDX lines in vitro (figure 1A). In a 10-day antigen-independent in vitro assay, cytoTIL15 persisted at greater frequencies than conventional TILs in the absence of IL-2 (figure 1B; *p<0.05). cytoTIL15 adoptively transferred into naïve NSG mice demonstrated ACZ-dependent long-term persistence without antigen or exogenous IL-2, whereas conventional TILs were undetectable $>30$ days following adoptive cell transfer (figure 1C). Importantly, cytoTIL15 achieved significant tumor control in a human PDX model (figure 1D), which correlated with increased TIL accumulation in secondary lymphoid organs.

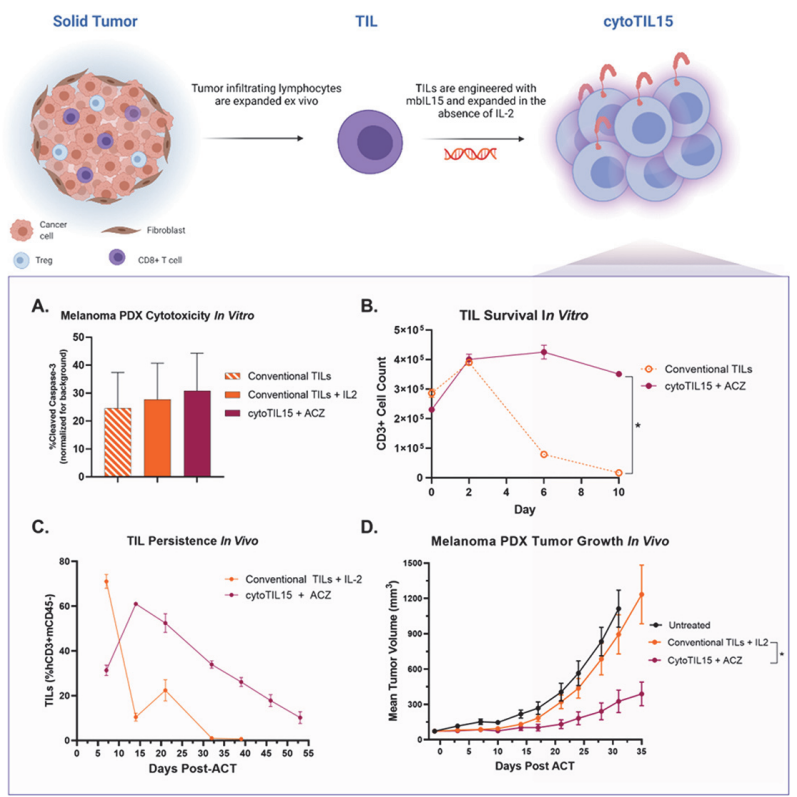

Abstract 166 Figure 1 cytoTIL15 demonstrate superior persistence. cytoTIL15 is an engineered TIL product expressing regulatable mbIL15. (A) cytoTIL15 demonstrate enhanced in vitro cytotoxicity after co-culture with melanoma tumor lines (representative data from 3 TIL donors). (B) cytoTIL15 have improved persistence in antigen- and IL2- independent culture conditions in vitro compared to conventional TILs cultured in the absence of IL-2 as well as (C) in vivo compared to conventional TILs supplemented with IL-2, when engrafted into NSG mice (in vitro: representative data from 1 TIL donor, performed in $>3$ replicate donors, in vivo: $n=5 /$ group, representative of 1 TIL donor, performed in $>3$ replicate donors). (D) cytoTIL15 (with 200mg/kg ACZ PO QD) demonstrate enhanced anti-tumor efficacy in a xenograft melanoma model as compared to conventional TILs (with 50000 IU IL-2 q8h BID, IP for 5 days) ( $n=8 /$ group, representative of 1 TIL donor, performed in $>2$ replicate donors; $\mathrm{ACT}=$ adoptive cell transfer).

Conclusions Taken together, the superior persistence and potency of cytoTIL15 in the complete absence of IL-2 highlights the clinical potential of cytoTIL15 as a novel TIL product with enhanced safety and efficacy for patients with melanomas, and other solid tumors.

Acknowledgements The authors wish to acknowledge the Cooperative Human Tissue Network for the their supply of human tumor tissue, and the MD Anderson Cancer Center for technical support; schematic created with BioRender.com.

\section{REFERENCES}

1. Chandran SS, Somerville RPT, Yang JC, Sherry RM, Klebanoff CA, Goff SL, Wunderlich JR, Danforth DN, Zlott D, Paria BC, Sabesan AC, Srivastava AK, Xi L, Pham TH, Raffeld M, White DE, Toomey MA, Rosenberg SA, Kammula US. Treatment of metastatic uveal melanoma with adoptive transfer of tumour-infiltrating lymphocytes: a single-centre, two-stage, single-arm, phase 2 study. Lancet Oncol 2017 Jun;18(6):792-802. doi: 10.1016/S1470-2045(17)30251-6. Epub 2017 Apr 7. PMID: 28395880; PMCID: PMC5490083.

2. Yang JC. Toxicities associated with adoptive T-cell transfer for Cancer. Cancer $J$ 2015;21:506-9.

3. Schwartz RN, Stover L, Dutcher JP. Managing toxicities of high-dose interleukin-2 Oncology (Williston Park) 2002 Nov;16(11 Suppl 13):11-20. PMID: 12469935.

http://dx.doi.org/10.1136/jitc-2021-SITC2021.166 